\title{
Komplexität beherrschen
}

Liebe Leserin, lieber Leser,

die Randbedingungen bei der Entwicklung von Antrieben haben sich in den vergangenen Jahren massiv verändert. Beim konventionellen Verbrennungsmotor steigt die Anzahl an Applikationsvarianten eines Motors, die technisch beherrscht und zu akzeptablen Kosten dargestellt werden müssen, ständig an. So kam beispielsweise Mercedes-Benz Anfang der 1990er-Jahre beim Vierzylinder-Pkw-Dieselmotor noch mit circa 70 Varianten aus, um weltweit alle gesetzlichen und kundenseitigen Anforderungen zu erfüllen. Ende der 1990er-Jahre stieg die Zahl schon auf rund 200, heute sind es bei der aktuellen Version sogar etwa 1000 Varianten, die unter anderem durch weltweit strengere Emissions- und $\mathrm{CO}_{2}$ Richtlinien zustande kommen. Parallel entwickeln Automobilhersteller und Zulieferer mit großem finanziellen und personellen Aufwand unterschiedlichste alternative Antriebskonzepte zur Serienreife, um Lösungen für die Mobilitätsanforderungen von morgen zu bieten.

Mit den neuen Aufgaben wird auch der Entwicklungsprozess zunehmend komplexer. Die zentrale Frage dabei ist, mit welchen Methoden und Werkzeugen er auch künftig optimal gemanagt werden kann. Eine Antwort ist der offensichtliche Trend, die Entwicklungsleistung vom Fahrversuch zum Prüfstand und von dort in die Simulation zu verlagern. Ermöglicht wird dies unter anderem durch leistungsfähigere Hardware und Simulationssoftware, die die Realität immer besser widerspiegelt. Aber auch die Prüfstandstechnik hat sich in letzter Zeit stark weiterentwickelt, beispielsweise um dynamische Fahrvorgänge für RDE-Untersuchungen oder das thermische Aufheizverhalten der Abgasnachbehandlung für Kaltstartbetrachtungen besser abbilden zu können.

Mit diesem ATZextra möchten wir Ihnen einen Überblick über neue Entwicklungen bei Prüfständen und Simulationen für Antriebe geben. Denn künftige Antriebsstrang-Entwicklungsaufgaben sind nur mit einem umfassenden Einsatz von Simulationen und Prüfstandsuntersuchungen zu stemmen. Nur mit ihrer Hilfe können Verbrennungsmotoren wirtschaftlich weiterentwickelt und gleichzeitig alternative Antriebe zur Großserienreife gebracht werden.

Herzlichst, Ihr

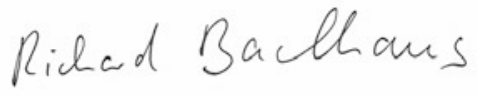

Richard Backhaus Korrespondent ATZextra

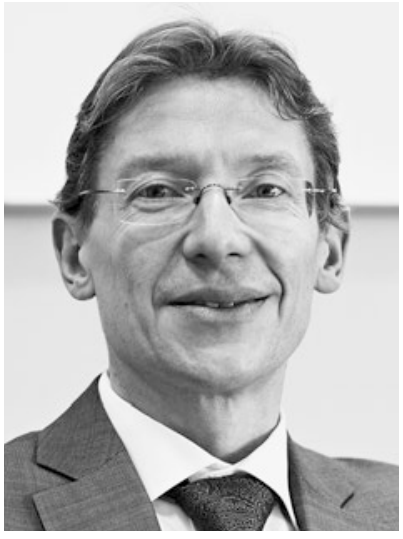

Wir entwickeln, was bewegt

\section{IAV - Ihr Partner \\ für Automotive Engineering}

